\title{
Harnessing Non-Uniform Transmit Power Levels for Improved Sequence Based Localization
}

\author{
Suvil Deora \\ Department of Electrical Engineering \\ University of Southern California \\ Los Angeles, USA \\ Email: deora@usc.edu
}

\author{
Bhaskar Krishnamachari \\ Department of Electrical Engineering \\ University of Southern California \\ Los Angeles, USA \\ Email: bkrishna@usc.edu
}

\begin{abstract}
Sequence-based localization (SBL) is a technique whereby a node is localized based on the ranked sequence of signal strengths obtained from a set of beacon nodes. SBL effectively partitions the area into regions corresponding to unique ranked sequences. Prior work has developed SBL under the assumption that all beacons have the same transmit power. In this work, we consider beacons with unequal transmit power for sequencebased localization and present heuristic algorithms for joint transmit power optimization and beacon placement. We show through comprehensive simulations that a novel enhancement of SBL utilizing optimized non-uniform transmit powers, coupled with careful beacon placement, which we refer to as NU-SBL, can dramatically improve the area partitioning compared to traditional SBL. However, in evaluating these schemes under stochastic fading, we find that the original SBL with optimized location performs nearly as well or slightly better than NU-SBL in many cases. We introduce another scheme, that we refer to as NU-SBL-ZOOM, which further allows the power levels to be optimized non-uniformly so as to focus in on a particular smaller region within the larger localization space. NU-SBL-ZOOM is found to perform much better in terms of both area partitioning as well as location error in the presence of fading.
\end{abstract}

\section{INTRODUCTION}

Indoor localization using existing Wi-Fi infrastructure or embedded wireless sensors can be seen as an important building block for a vast array of commercial applications involving humans with smart phones or tracking of industrial assets. It is desired to have good localization accuracy without any additional infrastructure cost. Numerous techniques such as fingerprinting [1] [2], least squares estimation [3], maximum likelihood estimation (MLE) [4], and sequence-based localization (SBL) [5] have been developed and deployed to achieve this goal. In all these techniques, Received Signal Strength (RSS) from the neighboring access points (beacons) is used to deduce the position of the receiver. We are interested in exploring how RSS based localization can be further improved by carefully tuning and placing existing access points without increasing their cost.

One of the programmable settings on an access point is the transmit power, and it may in many cases also be possible to determine its placement in the environment. We show that these play a vital role: it is to improve location accuracy substantially by carefully placing and assigning transmit power to beacons.

Motivation: Our work is motivated by our experience with implementing a real-world indoor localization system at
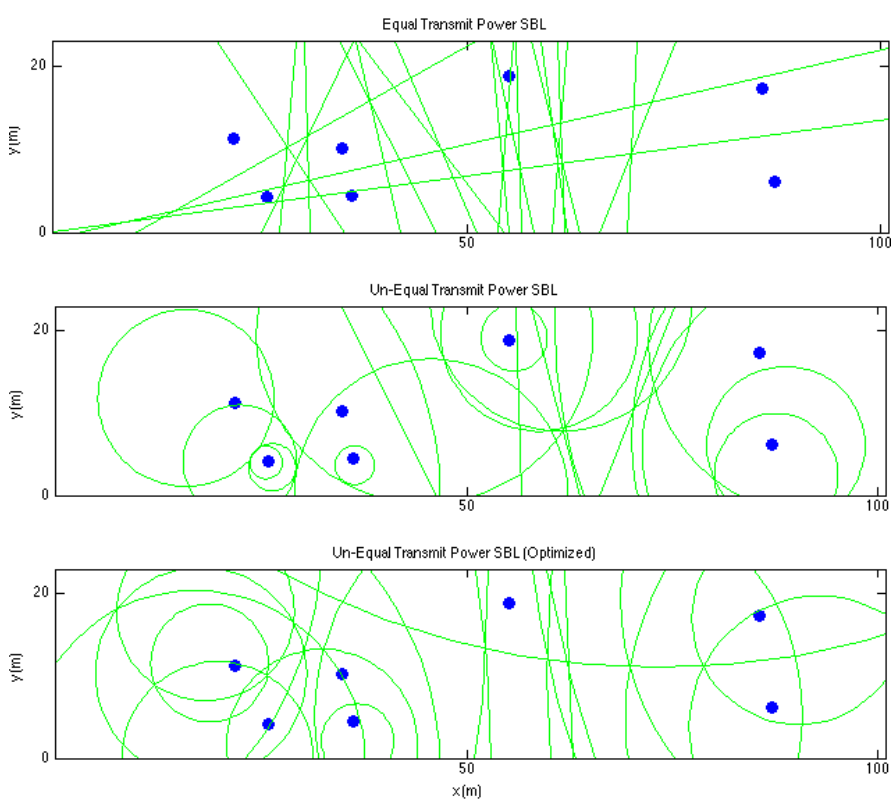

Fig. 1. Area Partitioning for Sequence-based Localization under (a) Equal Transmit Powers, (b) Real Power Settings, and (c) Optimized Power Settings from WiFi access points located in a real office building.

the USC Cinema School to enable personalized interactive media. We developed the system based on sequence-based localization [5], with pre-deployed WiFi access points acting as beacons and users carrying mobile devices in the space being located. The mobile devices collected RSS readings from each AP, and sent them to a central server where the SBL computations were performed. In traditional SBL, it is assumed that all beacons transmit at the same power, and the area is partitioned into regions based on linear perpendicular bisectors between pairs of beacons that represent equal RSS from both beacons. Each region then corresponds to a unique RSS sequence, which is used to identify the location of the unknown nodes. For the building where we had deployed our system, this partition of the area via equal-RSS lines is shown in Fig 1(a). However, we found that in practice AP's are deployed with significantly different transmit powers. This motivated us to determine the correct area partitioning with unequal powers for sequence-based localization, as shown in Fig 1(b). As can be seen, when the transmit powers are unequal, the curves separating different sequences, which we 


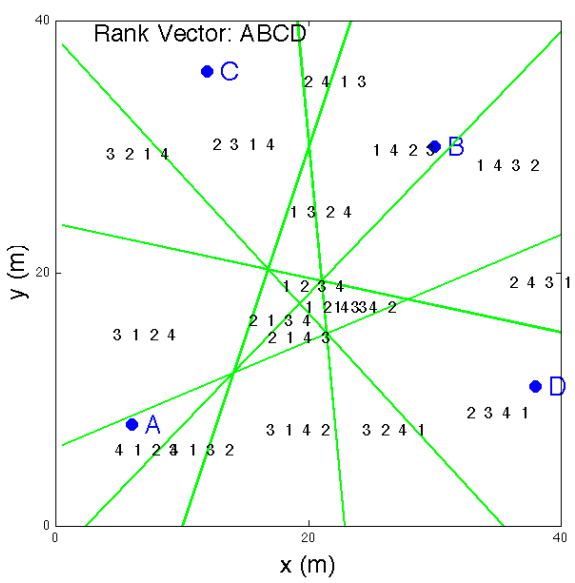

(a)

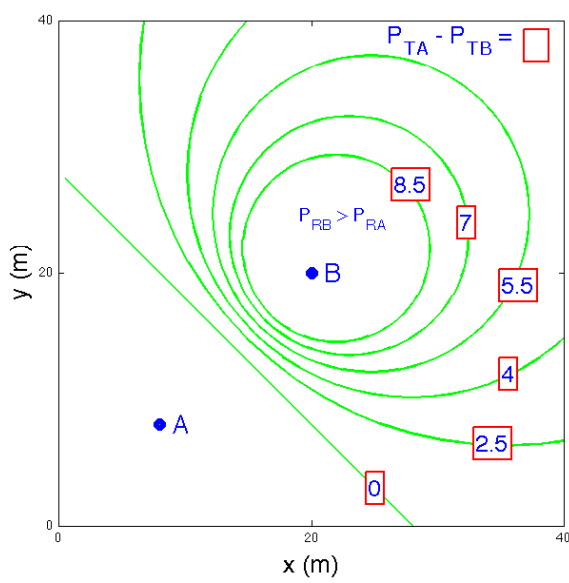

(b)

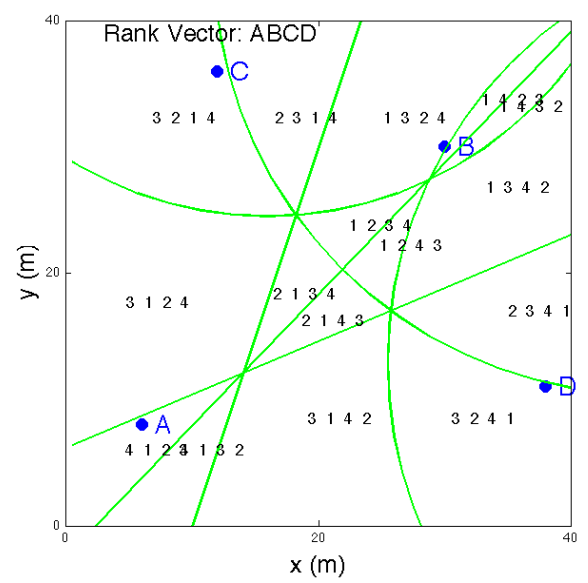

(c)

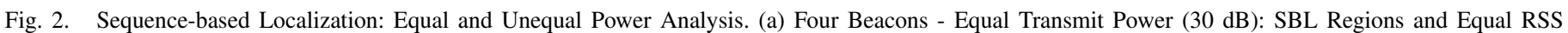

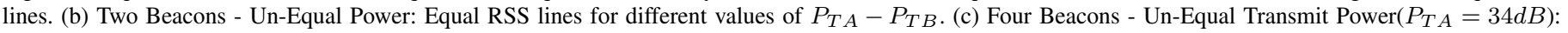
SBL Regions and Equal RSS lines.

refer to as equal-RSS curves, are no longer straight lines, but rather circles (proved later in this paper). This got us thinking about the possibility of using the transmit powers as a design variable to try and create more equitable partitions with the aim of reducing the localization error. Fig 1(c) shows that for this particular deployment of access points, optimizing the transmit power can result in a configuration of equal-RSS curves such that the area of the largest region is reduced all the way to $92.82 \mathrm{~m}^{2}$ (from $487.4 \mathrm{~m}^{2}$ obtained in the case of equal powerbased area partition, and $316.6 \mathrm{~m}^{2}$ obtained in case of the observed unequal powers).

Motivated by this experience, we design and evaluate nonuniform transmit power based SBL (NU-SBL) in this paper, making the following contributions:

- We derive the area partitioning approach for SBL with non-uniform powers (NU-SBL), which has not been considered before.

- We determine a mathematical bound on the number of faces that result from NU-SBL partition. This gives an intuition about the average area size reduction using NU-SBL compared to the previously considered equal powers SBL.

- We use simulated annealing to systematically evaluate the worst-case area partitioning improvement obtained by NU-SBL compared to SBL, when only the transmit powers of beacons are optimized with fixed locations.

- We then systematically evaluate the worst-case area improvement obtained by NU-SBL when both the transmit powers and the location of the beacons are optimized jointly. We show that this results in an even bigger improvement.

- We further present NU-SBL-ZOOM, a novel "zoom" enhancement for NU-SBL, which allows for optimizing the transmit powers to locate individuals in a sub-portion of the full region. The area partitioning improvement obtained by this approach are found to be quite dramatic.
- We then undertake simulations that take into account realistic stochastic fading (with a log-normal distribution). Varying both the path loss exponents and the standard-deviation of fading, we compare the worstcase average error performance of traditional SBL with grid placement, SBL with optimized placement, NU-SBL, Least squares estimation, and NU-SBLZoom.

Our experiments with realistic fading show that traditional SBL is improved by careful placement of beacons, and in fact is about as good as or even better than NU-SBL in which both placement and powers are optimized for best area partitioning. However, NU-SBL-ZOOM, is shown to perform significantly better. All schemes outperform least-squares estimation at higher levels of fading.

The rest of the paper is organized as follows. We begin by recalling how SBL works, and how it can be extended with non-uniform transmit powers, including some basic analysis in Sections 2, 3, 4. We then present various optimizations and approaches for non-uniform SBL, evaluating them initially purely in terms of area partition in Sections 5 and 6. We then evaluate the proposed approaches under stochastic fading in 7 and describing related work in section 8. Finally, we draw conclusions and identify future directions in Section 8.

\section{SEQUENCE BASED LocAlizATION}

Sequence Based Localization, first presented in [5], works on partitioning the entire localization space into regions based on the received signal strength ranked vectors. Consider two beacons $A$ and $B$ located in a 2D space as shown in Fig 2(b), the entire space can be divided into two distinct regions separated by equal-RSS locus: the set of points such that a receiver located at any of these points will read equal RSS value from both $A$ and $B$. If the two beacons have equal transmit power then the equal-RSS locus is the perpendicular line bisector of the line joining the two beacons as shown in the Fig 2(b) for $P_{T B}-P_{T A}=0$ case. Each of the two regions have a unique rank vector. In a general setting with $n$ beacons there will be $\left(\begin{array}{l}n \\ 2\end{array}\right)$ perpendicular bisectors, each of 
which divides the space into two regions. Consider the faces (polygonal regions) created by the superposition of these lines in 2D space, Fig 2(a). Each such face corresponds to a unique set of rank ordering over all beacons. There can be $O\left(n^{n}\right)$ possible rank vectors out of which only $O\left(n^{4}\right)$ are feasible because the total possible number of faces are $O\left(n^{4}\right)$ [5]. In an ideal world a receiver node will generate a feasible ranked vector by measuring the signal strengths and hence localize its position to one of these faces. But due to fading, shadowing and other random, non-linear effects the generated vectors may or may not be one of the feasible ones. To overcome this SBL utilizes Kendall Tau distance as a metric to find the closest feasible vector and localize the position of the target node to the corresponding face.

Now if we consider two beacons with unequal transmit power, the equal-RSS locus is no more the perpendicular bisector of the line joining the two beacons. The shape and position of locus is strongly dependent upon the difference in transmit powers and the path loss exponent of the environment. For a two node system the equal-RSS locus for different values of $P_{T B}-P_{T A}$ are shown in Fig 2(b), as we increase the transmit power of one of the nodes the equal-RSS locus turns into a circle and shrinks around the beacon with smaller transmit power. The entire 2D space is still divided into two faces, but the noticeable difference from the equal power case is that now one of these faces is closed. Even with the modified shape of the equal-RSS locus it is still possible to use the same principles for localization. In general for $n$ beacons there will be $\left(\begin{array}{l}n \\ 2\end{array}\right)$ equal RSS locus, which divide the 2D space into $O\left(n^{4}\right)$ number of faces. This control over the shape of the equal RSS loci gives an opportunity to increase the number of faces in the localization space, which correlates with the accuracy of the algorithm as each face gets smaller on average. Fig 2(a), 2(c) shows how for a four node $(\mathrm{A}, \mathrm{B}, \mathrm{C}, \mathrm{D})$ topology the faces modify as we increase the $P_{T A}$ of beacon $A$ keeping the others same.

\section{AREA PARTITIONING FOR NU-SBL}

For regular SBL with equal powers it easy to see that the equal RSS locus between two beacons is their perpendicular bisector; here we derive the equal RSS locus in case of unequal powers.

Say a point $Q(x, y)$ is located at $d_{1}$ and $d_{2}$ distance from two beacons $A\left(x_{1}, y_{1}\right)$ and $B\left(x_{2}, y_{2}\right)$ then

$$
\begin{aligned}
& d_{1}=\sqrt{\left(x-x_{1}\right)^{2}+\left(y-y_{1}\right)^{2}} \\
& d_{2}=\sqrt{\left(x-x_{2}\right)^{2}+\left(y-y_{2}\right)^{2}}
\end{aligned}
$$

If beacon $A$ and $B$ are transmitting at un-equal power $P_{T A}$ and $P_{T B}$ respectively. According to the path-loss model for signal propagation, the RSS values at point $Q$ due to node $A$ and $B$ are given by:

$$
\begin{aligned}
& P_{R A}=P_{T A}-P_{d_{0}}-10 \eta \log \left(d_{1} / d_{0}\right) \\
& P_{R B}=P_{T B}-P_{d_{0}}-10 \eta \log \left(d_{2} / d_{0}\right)
\end{aligned}
$$

By equating $P_{R A}$ and $P_{R B}$ we can find the set of points where the received power is equal.

$$
P_{T A}-10 \eta \log \left(d_{1}\right)=P_{T B}-10 \eta \log \left(d_{2}\right)
$$

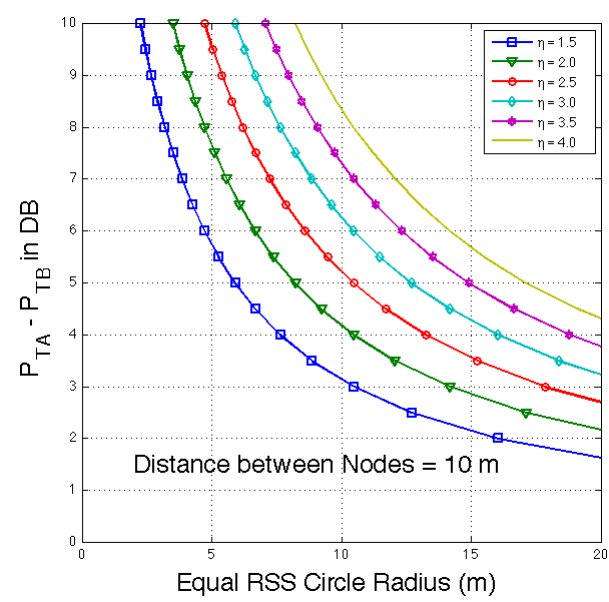

Fig. 3. Difference in Transmit Power vs Equal-RSS circle radius

without loss of generality we can assume $d_{2}>0$

$$
P_{T A}-P_{T B}=10 \eta \log \left(d_{1} / d_{2}\right)
$$

Let

$$
10^{\frac{P_{T A}-P_{T B}}{10}}=k
$$

Where $k$ is a positive constant. Substituting $d_{1}, d_{2}$ based on equations (1) and (2) and solving, we get

$$
\begin{array}{r}
\left(1-k^{\frac{2}{\eta}}\right) x^{2}+\left(1-k^{\frac{2}{\eta}}\right) y^{2}-2 x\left(x_{1}-k^{\frac{2}{\eta}} x_{2}\right) \\
-2 y\left(y_{1}-k^{\frac{2}{\eta}} y_{2}\right)+x_{1}^{2}+k^{\frac{4}{\eta}} x_{2}^{2}+y_{1}^{2}+k^{\frac{4}{\eta}} y_{2}^{2}=0
\end{array}
$$

Comparing with the standard non-degenerate conic section equation

$$
A x^{2}+B x y+C y^{2}+D x+E y+F=0
$$

we get

$$
A=\left(1-k^{\frac{2}{\eta}}\right), B=0, C=\left(1-k^{\frac{2}{\eta}}\right)
$$

hence $B^{2}-4 A C=-4\left(1-k^{\frac{2}{\eta}}\right)^{2}<0$ and $A=C$ which makes this curve a Circle.

The center of this circle is always on the line which passes through beacons $A$ and $B$. As the difference in transmit power $P_{T A}-P_{T B}$ increases, the center moves from $\infty$ towards beacon $B$ never crossing it. Also the radius of this circle shrinks to zero as the $P_{T A}-P_{T B}$ becomes larger and larger. It is interesting to see how the radius of this circle changes with $P_{T A}-P_{T B}$ and $\eta$. This can be seen in Fig 3. From the Fig 3 we see that if we want a circle with $\infty$ radius we can use equal transmit power for both the beacons for any value of $\eta$. On the other hand if we want a smaller radius circle we would need to increase the value of $P_{T A}-P_{T B}$. Another observation is that for the same radius and a higher $\eta$ we would need a higher $P_{T A}-P_{T B}$.

\section{MAXIMUM NUMBER OF FACES}

For $n$ beacons in the localization space, the maximum number of faces is $O\left(n^{4}\right)$ each one of which corresponds to unique ranked RSS vector. One of the properties of the ranked vectors for faces is that all the elements are unique. Therefore out of $n$ ! possible vectors only $O\left(n^{4}\right)$ are feasible face vectors. 


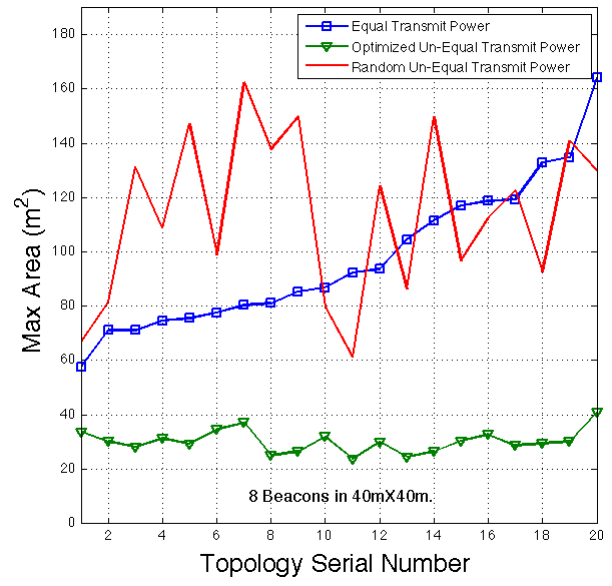

Fig. 4. Max-Area improvement by power optimization for 20 randomly picked topologies.

The upper bound on number of faces for equal power case is given by $\frac{n^{4}}{8}-\frac{5 n^{3}}{12}+\frac{7 n^{2}}{8}-\frac{7 n}{12}+1[5]$.

We hereby derive similar upper bound for the unequalpowers case. Let $f_{m}$ be the maximum number of faces generated by intersection of $\mathrm{m}$ circles. For $m=1, f_{m}=2$. Now let us assume that there are $m-1$ circles and we add one more. The new circle will intersect each of the existing circles at maximum 2 points. Hence the $m^{t h}$ circle can have $2(m-1)$ intersection points with rest of the $m-1$ circles. The $2(m-1)$ intersection points divide $m^{t h}$ circle into $2(m-1)$ curve segments. Each of which can potentially split an existing face in two, adding $2(m-1)$ more faces to the existing face count. This can be written as a recursion.

$$
\begin{aligned}
& f_{m}=f_{m-1}+2(m-1) \\
& \Rightarrow f_{m}=(m-1) m+2
\end{aligned}
$$

The above proof holds true for any set of convex closed curves. Now we know that $n$ beacons will have $\frac{n(n-1)}{2}$ equal-RSS circles. Therefore maximum number of faces $\stackrel{2}{F}_{m}$ will be given by

$$
F_{n}=f_{\frac{n(n-1)}{2}}=\frac{n^{4}}{4}-\frac{n^{3}}{2}-\frac{n^{2}}{4}+\frac{n}{2}+2, n>1
$$

Comparing the $F_{n}$ upper bounds for equal and unequal power we see that for that for the same number of beacons operating at unequal power can potentially generate more number of faces. As we discussed earlier higher number of faces correspond to higher accuracy.

\section{NU-SBL OPTIMIZATIONS}

In this section we discuss two main flavors of NU-SBL optimizations for the entire localization space. First we optimize over the transmit power of beacons; this is important from the pre-existing static beacon deployment point of view. Second, we look at the optimization over power as well as location. SBL and NU-SBL being inherently a geometry dependent scheme, this extra degree of freedom can significantly improve the algorithmic accuracy.
TABLE I. OPTIMIZATION IMPROVEMENTS

\begin{tabular}{|c|c|c|c|c|}
\hline$N$ & $\begin{array}{c}\text { Regular SBL } \\
\text { Grid Topology }\end{array}$ & $\begin{array}{c}\text { Power Opt. for } \\
\text { Grid Topology }\end{array}$ & $\begin{array}{c}\text { Location Opt. } \\
\text { for Equal } \\
\text { Power }\end{array}$ & $\begin{array}{c}\text { Joint Power } \\
\text { and Location } \\
\text { Opt. }\end{array}$ \\
\hline 8 & $55.7 \mathrm{~m}^{2}$ & $33.15 \mathrm{~m}^{2}$ & $26.55 \mathrm{~m}^{2}$ & $15.4 \mathrm{~m}^{2}$ \\
\hline 16 & $16.9 \mathrm{~m}^{2}$ & $3.9 \mathrm{~m}^{2}$ & $3.6 \mathrm{~m}^{2}$ & $1.9 \mathrm{~m}^{2}$ \\
\hline
\end{tabular}

\section{A. NU-SBL with power optimization}

Here we present simulation results which show how for any given topology of beacons, we can improve the localization error by transmit power vector $\overrightarrow{P_{T}}$ optimization. The metric used for optimization is the area of the largest face generated by equal-RSS circles, let us call it Max-Area. We want to minimize Max-Area for a given topology this in-turn would minimize the worse case localization error bringing down the average error. This is not a convex optimization.

For this we picked 20 randomly chosen 8 beacon topologies in a $40 \mathrm{~m} X 40 \mathrm{~m}=1600 \mathrm{~m}^{2}$ area. We use simulated annealing for our optimization. We run several instances of simulated annealing for a given topology and pick optimum $\vec{P}_{T}{ }^{*}$ for the minimum Max-Area. These results are shown in Fig 4. The three plots represent Max-Area for equal transmit power $\overrightarrow{P_{T}}$, randomly chosen $\overrightarrow{P_{T}}$ and optimum ${\overrightarrow{P_{T}}}^{*}$. We observe that it is possible to significantly improve the localization accuracy for any given topology just by finding the right transmit power for each node. Also not all un-equal transmit power vectors $\overrightarrow{P_{T}}$ are not necessarily better. For getting this improvement we need to know the value of path loss exponent $\eta$. For the above experiment we used $\eta=2$, but the observation holds true for any value of $\eta$.

\section{B. NU-SBL: SBL with power and location optimization}

Learning from the power optimization work we decided to take it a step further and optimize over both location and power of a beacon jointly. The simulated annealing optimization was modified so as to be used in this scenario. Simulated annealing was chosen the way to optimize because of the computational complexity of the problem. For any other method, it was computationally not possible to converge for more then 4 nodes in $40 m \times 40 m=1600 m^{2}$ area. It must be reminded that these calculations are only needed once at the time of deployment and with simulated annealing it is possible to obtain a good approximation of the optimal result in a very reasonable time.

For this section only the number of beacons $N$, total area $A=40 \mathrm{~m} X 40 \mathrm{~m}=1600 \mathrm{~m}^{2}$ and $\eta=2.0$ are fixed. Fig 5, shows faces created by equal-RSS circles for regular SBL (radius $=\infty$ ) and all the other different flavors of optimization. For each sub-fig the corresponding value of MaxArea is also shown these results are also tabulated in table 1. Fig 5(a),5(b),5(c),5(h) are for $N=8$ and fig 5(e),5(f),5(g),5(h) are for $N=16$.

Fig 5(b), 5(f) show improvement by optimization over transmit power, while keeping the same grid topology used for regular SBL. Its is a significant improvement obtained by simply changing the each beacon's transmit power. Fig 5(c), $5(\mathrm{~g})$ explores the possibility of improvement by optimizing over the node locations while keeping equal transmit power for all beacons. The improvement is of the same order as 


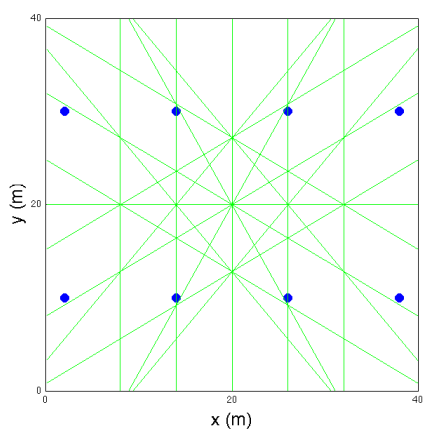

(a) Regular SBL Grid: $57.6 \mathrm{~m}^{2}$

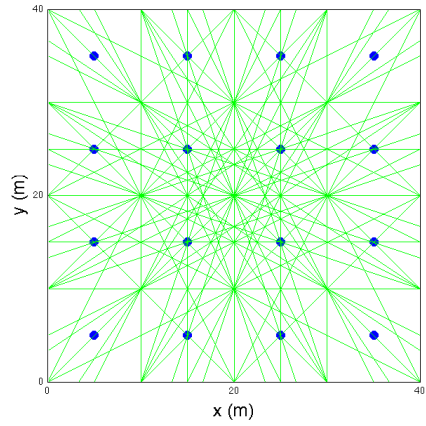

(e) Regular SBL Grid: $16.9 m^{2}$

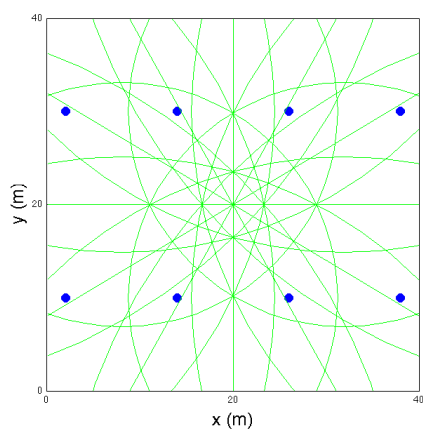

(b) $\operatorname{Opt}(\mathrm{Pwr}): 33.15 \mathrm{~m}^{2}$

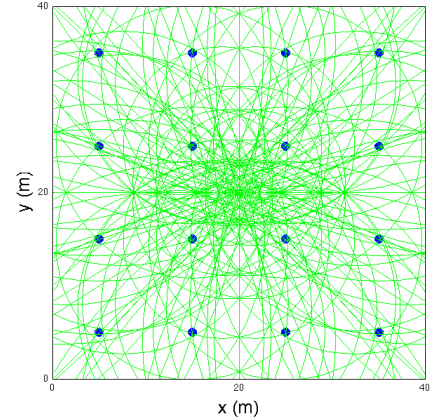

(f) $\operatorname{Opt}(\operatorname{Pwr}): 3.9 m^{2}$

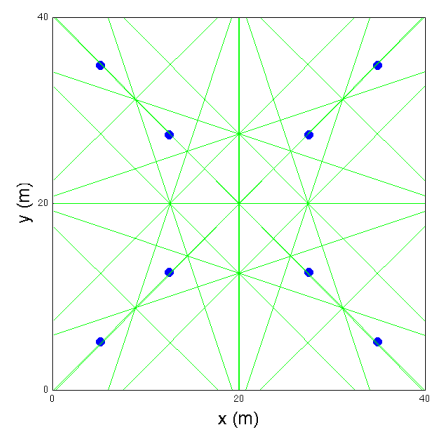

(c) $\operatorname{Opt}(\mathrm{X}, \mathrm{Y}): 26.55 \mathrm{~m}^{2}$

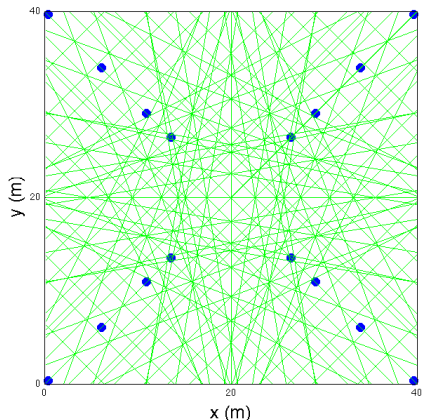

(g) $\operatorname{Opt}(\mathrm{X}, \mathrm{Y}): 3.6 m^{2}$

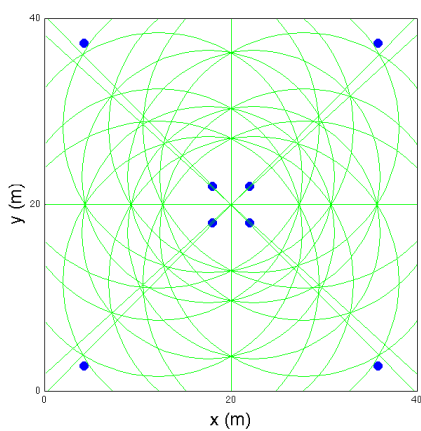

(d) $\operatorname{Opt}(\mathrm{X}, \mathrm{Y}, \mathrm{Pwr}): 15.4 \mathrm{~m}^{2}$

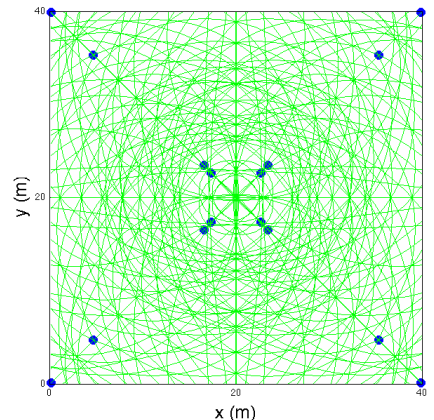

(h) $\operatorname{Opt}(\mathrm{X}, \mathrm{Y}, \mathrm{Pwr}): 1.9 \mathrm{~m}^{2}$

Fig. 5. Analyzing Power and Location Optimization for 8 and 16 Beacon Case. Objective: Minimize Max-Area. (a)(e) Grid Topology: No optimization. (b)(f) Grid Topology: Only Power optimization. (c)(g) Equal Power: Only Location optimization. (d)(h) Joint Location and Power optimization.

Fig 5(b),5(f). Next in Fig 5(d),5(h) we show how the joint optimization over location and power of beacons can significantly improve the theoretical accuracy of SBL. It can also be seen from the Fig 5(h),5(d) how the faces are uniformly distributed over the entire area and are of similar size. The Max-Area for $n=16$ plunges from $16.9 \mathrm{~m}^{2}$ to $1.9 \mathrm{~m}^{2}$ for the shown total area.

Following are the key observations from these simulations:

- Beacon locations and power are symmetric with respect to the center of the plane in case of OPT(XY) and OPT(XYPwr). Where OPT(XY) means optimized for location only and OPT(XYpwr) means jointly optimized for both transmit power and location.

- Optimized Beacon locations tend to be on $X=Y$ and $X=-Y$ if center of the plane is considered the origin.

- Our simulations suggest first optimizing for location assuming equal powers, and then optimizing for power doesn't yield any further improvement.

\section{NU-SBL wITH Zoom}

In real world scenarios people are not uniformly spread out inside a building. They tend to gather in certain areas. For example if there is a conference going on inside a building then relatively there well be a lot more people inside the conference room then anywhere else. So the question arises that can we selectively improve the localization accuracy in certain areas. The answer is yes and the solution is the proposed zoom technique.
Say we already have the optimized the location and power for all the beacons, this setting is represented in Fig 6(a) and now we want to improve localization accuracy in the $10 \mathrm{~m} \times 10 \mathrm{~m}$ box around the point $(5,5)$, we can do this by optimizing only the transmit power of the beacons for the same topology. For this optimization our metric should be the Max-Area for that specific $10 \mathrm{~m} \mathrm{X} 10 \mathrm{~m}$ Box. This scenario is shown in Fig 6(c). By doing this optimization we improve the accuracy in any part of the building just by changing the beacon transmit power vector $\overrightarrow{P_{T}}$. These vectors can be precomputed for different parts of the building.

Fig 6(b) shows another interesting scenario. Say we want to track an individual inside a building, we can use the same zoom technique just by decreasing the size of the box and moving it along with the individual by changing $\overrightarrow{P_{T}}$ every second. The results in Fig 6(d) show that Max-Area value drops to almost zero if we use this technique with a smaller area to focus. But even if the area is as large as $100 \mathrm{~m}^{2}$ the Max-Area values (which represent the worst case area size) are, remarkably, less then $0.5 m^{2}$.

\section{EVALUATION UNDER FADING}

In a real world setting, localization error for any RSS based scheme is highly dependent upon the RF-channel parameters. In this section we evaluate SBL (i.e., equal powers, with and without location optimization), NU-SBL, and ZOOM localization schemes in a real world like scenario, using extensive simulations. In our simulations we consider RSS variations caused due to fading and shadowing. To incorporate them in our simulation we use the combined path loss and shadowing model [6]. We also compare SBL, NU-SBL and 


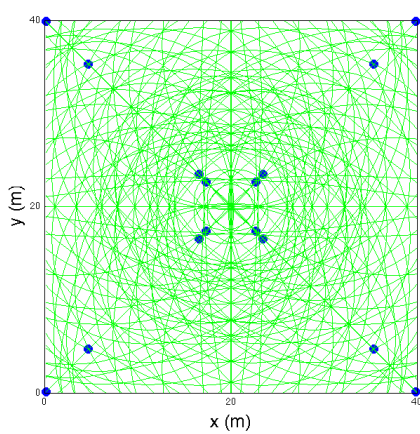

(a) Max-Area: $1.9 \mathrm{~m}^{2}$

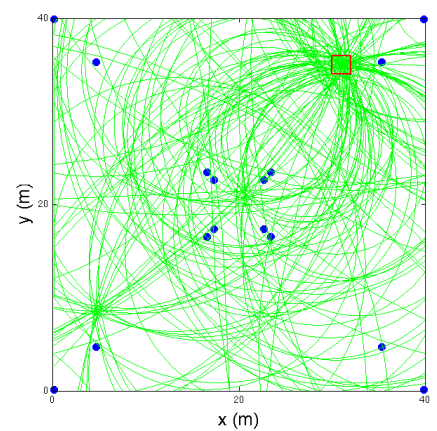

(b) Max-Area: $0.025 \mathrm{~m}^{2}$

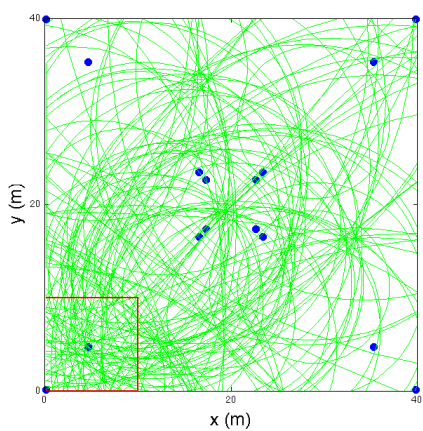

(c) Max-Area: $0.45 m^{2}$

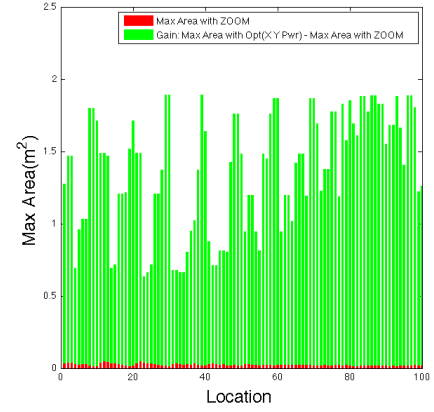

(d) Max-Area: $0.05 m^{2}$

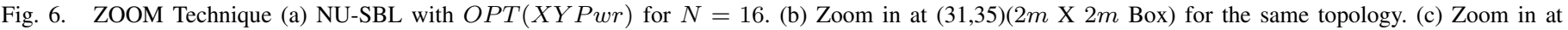
$(5,5)(10 m$ X $10 m$ Box) for the same topology. (d) Max-Area improvement with Zoom technique at different locations( $2 m$ X $2 m$ Box).

TABLE II. Simulation Parameters [7]

\begin{tabular}{|c|c|c|}
\hline Parameter & Typical Value & Typical Range \\
\hline$P_{d 0}$ & $37 d B\left(d_{0}=1 m\right)$ & NA \\
\hline$\eta$ & 2.2 & $1.5-3.5$ \\
\hline$\sigma$ & 6 & $4-10$ \\
\hline
\end{tabular}

TABLE III. RUN TIME COMPARISON $\left(n=16, A=1600 m^{2}\right)$

\begin{tabular}{|c|c|c|c|}
\hline & SBL & NU-SBL & LSE \\
\hline Worst case complexity & $\Theta\left(K n^{6}\right)$ & $\Theta\left(K n^{6}\right)$ & $\Theta(n A)$ \\
\hline Empirical Computation Time & $2-3 m S$ & $5-6 m S$ & $16-18 m S$ \\
\hline
\end{tabular}

ZOOM techniques with Least Square Estimator (LSE) scheme.

1) LSE: This scheme works by coming up with a distance estimate of the receiver from the $i^{\text {th }}$ beacon by using

$$
d_{R i}=10^{\frac{P_{T i}-P_{d 0}-P_{R i}}{10 \eta}}
$$

where $P_{R i}$ is the RSS vale from the $i^{t h}$ beacon. Then for each grid point in the localization space we calculate

$$
\Sigma_{(x, y)}=\sum_{i=1}^{N}\left(d_{R i}-d_{i}^{(x, y)}\right)^{2}
$$

where $d_{i}{ }^{(x, y)}$ is the euclidean distance of the $i_{t h}$ beacon from the $(x, y)$ grid point. The location estimate is given by $(x, y)$ for which $\Sigma_{(x, y)}$ is minimum. It must be noted that computational complexity of LSE is highly dependent on the total area of the localization space.

2) Simulation Model: One of the most widely used model for generating RSS values at a distance $d$ is the combined path loss and shadowing model [6]:

$$
P_{R}=P_{T}-P_{d_{0}}-10 \eta \log \left(d_{1} / d_{0}\right)-\psi_{\sigma}
$$

Where $P_{R}, P_{T}, P_{d_{0}}, \psi_{\sigma}$ are in $d B$ and $d, d_{0}$ are in $m$. This model is the superimposed version of path loss and non-linear effects due to fading and shadowing. Where $\psi_{\sigma}$ is a Gaussiandistributed random variable with zero mean and variance $\sigma^{2}$. Typical values of the equation parameters for an in-door WiFi environment are tabulated in table II. Our simulations evaluate the performance of different localization schemes for different values of path loss exponent $\eta$ and standard deviation $\sigma$.

3) Simulation Procedure: We have done all our simulations for 16 beacons placed in a $40 \mathrm{~m}$ X $40 \mathrm{~m}$ area. For regular SBL and LSE schemes we use a grid topology and equal transmit power as shown in Fig 5(e), note that there is no other more reasonable setting for LSE since at 0 variance it can provide an exactly correct solution regardless of beacon placement and none of the schemes are optimized for particular higher variances. For SBL, we also present results with the optimized location, which is independent of $\eta$. For NU-SBL we optimize for location and power for the given $\eta$ assuming zero fading and then use the resulting topology and transmit power settings for simulation at different fading levels. The NU-SBL-ZOOM10 scheme uses the same topology as NUSBL but has a different transmit power setting so as to focus on the $10 \mathrm{~m}$ X $10 \mathrm{~m}$ area around $(5,5)$. The NU-SBL-ZOOM2 scheme has a different transmit power setting so as to focus on the $2 m \times 2 m$ area around $(1,1)$.

Running the simulation involves generating the RSS vector using the above described simulation model for given $(\eta, \sigma)$ and then using the localization scheme to come up with the location estimate. We assume that noise affects $R S S_{i}$ value from the $i^{t h}$ beacon independently of the other RSS values. For every grid point under consideration we randomly generate 150 RSS vectors and compute the location estimate for each one of them to get the expected location error for that grid point with a $96 \%$ confidence interval. To generate the result graphs we pick a grid point with highest expected location error and plot its expected location error. This means that we are considering the worse case error over the entire space.

To be fair to the ZOOM techniques we only consider as the possible locations of the user being localized the grid points which are in the zoom region.

4) Evaluation Results: The results from the simulations are shown in Fig 7. The three cases presented are for different values of the path loss exponent $\eta$. (As a reference, note that the literature reports typical indoor path loss exponents of about 2.18 [7]).

- SBL with optimized location is singificantly better than SBL on a grid placement.

- $\quad$ surprisingly, NU-SBL with optimized location (with respect to area partitions) is only slightly better than SBL with optimized location for the no-fading case. But with fading, it turns out that optimized-locationSBL is more robust. 


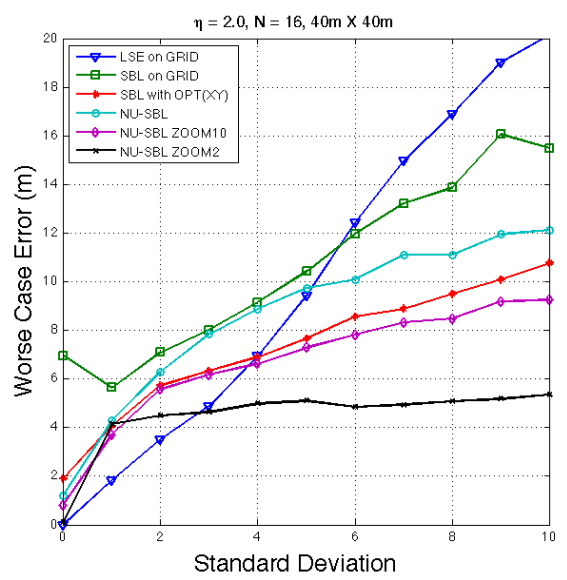

(a)

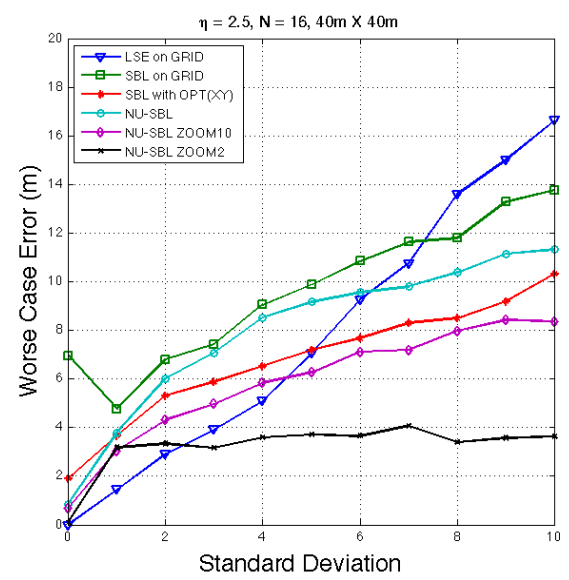

(b)

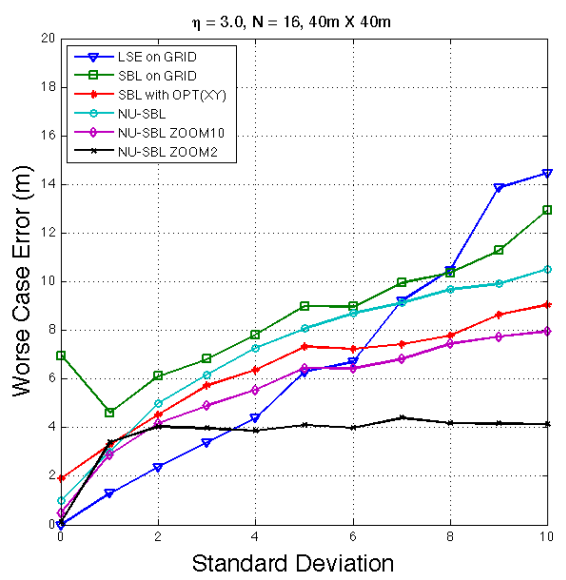

(c)

Fig. 7. Comparison of worse case location error when using LSE, SBL,SBL with optimized location, NU-SBL, NU-SBL-ZOOM as a function of $\sigma$. (a) $\eta=2.0$ (b) $\eta=2.5$ (c) $\eta=3.0$

- ZOOM technique improves NU-SBL significantly, and the ZOOM technique gives better results if the zoom area is smaller.

- $\quad$ SBL with optimized location, NU-SBL, NU-SBL with ZOOM all perform better then LSE at reasonable values of $\sigma$. The crosover point is between 2 and 5. This is an important comparison point for these schemes as LSE also requires the same information as NU-SBL (the path loss exponent) and performs arbitrarily well at low variances, limited only by the granularity at which space is sampled.

Table III compares the worst-case computational complexity and the empirically measured computational run-times for our implementations of the three techniques. It can be seen that NU-SBL takes about twice as long as SBL, but both are quite a bit faster than LSE for the simulated scenario. This is essentially because the compute time for LSE is directly tied to the size of the entire region, while the complexity of SBL schemes is a function of the number of beacons, and for typical densities of dozens of nodes is likely to be lower.

\section{RELATED WORK}

RF signal based indoor localization systems can be broadly categorized into RSS based systems, time of arrival [8] [9] and angle of arrival [10] based systems, and radio interferometry [11] [12] based systems. Propagation time and angle of arrival based systems have a few drawbacks in an indoor environment. Indoors it is difficult to have a line of sight channel and the RF propagation may suffer from multipath effect, which may seriously alter the time and angle of arrival of the signal, hence adversely affecting the accuracy. This issue is mitigated in ultra-wideband systems, however these schemes typically require more sophisticated transceivers and also require fine-grained global time synchronization, and consequently the hardware complexity and cost is higher, preventing them from being deployed easily in commodity radio platforms such as mobile phones and low power wireless sensors. Radio interferometry based localization works best with specialized tone-emitting radios [11] (though recent work has implemented them in other radios at the cost of reduced accuracy [13]) and works best in line of sight settings. It also requires tight synchronization and calibration and is computationally quite demanding due to the complexity of signal processing associated with it.

The RSS based localization systems are more robust and relatively less expensive to deploy in similar situations. The most basic technique in the literature that uses RSS is the proximity [14] based scheme. Where RSS value is quantized in 1 or 0 and the location of receiver is approximated as the nearest transmitter's location. RADAR [1] and LANDMARC [2] are examples of finger-printing based techniques that selects $k$ known locations from a database (developed using preliminary measurements) whose recorded RSS signature is closest to the one obtained by the receiver. Receiver location is obtained by averaging over the $k$ co-ordinates. We compare our techniques in this paper with the classical approach of least squares estimation (LSE) [3]. While LSE requires little prior training or estimation (other than the path loss exponent), as our results in this work demonstrate, it performs best under low fading variance. The high-performance, most sophisticated approach to pure RSS-based localization is maximum likelihood estimation [4]; however it requires an online, accurate, estimation of the fading variance and other channel parameters, and incurs a high algorithmic complexity.

Sequence Based Localization (SBL), first introduced as ecolocation in [15] and refined in [5], offers a low-complexity alternative to these approaches that requires no prior training or model estimation. It has therefore been found particularly well suited for emergency response operations where the localization system needs to be deployed rapidly [16]. One third party study [17] compared finger-printing, MLE and sequence based localization empirically under different antenna orientation and calibration settings. It showed that SBL can outperform the other approaches in an uncalibrated, dynamically-varying realistic environment, where poor estimation of model parameters or the underlying environment hurt the performance of MLE and fingerprinting approaches. Authors of [18] use series of sequences for improved tracking and in [19] they show how sequences can be used to find distance between neighbours. 
There has been little prior work on exploring tunable transmission powers for improving localization. One exception is a recent work on optimizing the power levels of beacons to improve location performance of ultra-wideband ToA based localization schemes while minimizing energy consumption [20].

Recent work has shown that RSS based localization techniques can be further enhanced in mobile-based localization systems by combining with readings from other sensors such as accelerometers and gyros, and taking into account the environment map [21]. Our work exploring enhancements of SBL aimed at improving low-complexity RSS localization are orthogonal and complementary to these efforts.

\section{CONClusions AND Future WORK}

We have carefully studied enhancements to sequence-based localization, one of the state of the art techniques for pure RF-based localization. The main contributions of this work have been to show that sequence based localization can be substantially improved by carefully optimized placement of beacons. We found that while the further optimization of power levels (in NU-SBL) brings improvements in area partitioning (ignoring fading), this improvement does not hold when considering stochastic fading. However, the novel zoom technique utilizing non-uniform powers in order to help localize a node known to be within a sub-region dramatically improves performance even with fading. We also compared these techniques with the more traditional least squares estimation approach and found that they offer benefits beyond certain thresholds of the fading standard deviation, ranging from 2 to 5 , and are also computationally faster by $3 x-6 x$.

While this work has made a head-start on understanding how non-uniform power levels can be used to improve localization accuracy, it does not offer insights on how knowledge of obstructions or real environment maps could be taken into account. In future work, we plan to undertake more sophisticated algorithm design and a more detailed evaluation in realistic environments that take into account path loss due to walls in simulations based on real building maps as well as in real deployments. This will be a non-trivial extension as it requires further work to consider the additional non-linearities in the area partitioning introduced by walls; real deployments may also offer more data-driven ways to determine the partitioning rather than the model-based approach used in this work, which assumes that the path loss exponent is homogeneous throughout the environment. Also developing a technique with provable approximation may be of interest.

\section{REFERENCES}

[1] P. Bahl and V. Padmanabhan, "Radar: an in-building rf-based user location and tracking system," in INFOCOM 2000. Nineteenth Annual Joint Conference of the IEEE Computer and Communications Societies. Proceedings. IEEE, vol. 2, 2000, pp. 775-784 vol.2.

[2] L. Ni, Y. Liu, Y. C. Lau, and A. Patil, "Landmarc: indoor location sensing using active rfid," in Pervasive Computing and Communications, 2003. (PerCom 2003). Proceedings of the First IEEE International Conference on, March 2003, pp. 407-415.

[3] A. Savvides, C.-C. Han, and M. B. Strivastava, "Dynamic fine-grained localization in ad-hoc networks of sensors," in Proceedings of the 7th Annual International Conference on Mobile Computing and Networking, ser. MobiCom '01. New York, NY, USA: ACM, 2001, pp. 166179
[4] A. Waadt, C. Kocks, S. Wang, G. Bruck, and P. Jung, "Maximum likelihood localization estimation based on received signal strength," in Applied Sciences in Biomedical and Communication Technologies (ISABEL), 2010 3rd International Symposium on, Nov 2010, pp. 1-5.

[5] K. Yedavalli and B. Krishnamachari, "Sequence-based localization in wireless sensor networks," Mobile Computing, IEEE Transactions on, vol. 7, no. 1, pp. 81-94, Jan 2008.

[6] A. Goldsmith, Wireless communications. Cambridge university press, 2005.

[7] E. Tanghe, W. Joseph, L. Verloock, L. Martens, H. Capoen, K. Van Herwegen, and W. Vantomme, "The industrial indoor channel: largescale and temporal fading at 900, 2400, and $5200 \mathrm{mhz}$," Wireless Communications, IEEE Transactions on, vol. 7, no. 7, pp. 2740-2751, July 2008.

[8] S. Gezici, Z. Tian, G. Giannakis, H. Kobayashi, A. Molisch, H. Poor, and Z. Sahinoglu, "Localization via ultra-wideband radios: a look at positioning aspects for future sensor networks," Signal Processing Magazine, IEEE, vol. 22, no. 4, pp. 70-84, July 2005.

[9] R. Fontana, E. Richley, and J. Barney, "Commercialization of an ultra wideband precision asset location system," in Ultra Wideband Systems and Technologies, 2003 IEEE Conference on, Nov 2003, pp. 369-373.

[10] B. Van Veen and K. Buckley, "Beamforming: a versatile approach to spatial filtering," ASSP Magazine, IEEE, vol. 5, no. 2, pp. 4-24, April 1988.

[11] M. Maróti, P. Völgyesi, S. Dóra, B. Kusý, A. Nádas, A. Lédeczi, G. Balogh, and K. Molnár, "Radio interferometric geolocation," in Proceedings of the 3rd International Conference on Embedded Networked Sensor Systems, ser. SenSys '05. New York, NY, USA: ACM, 2005, pp. $1-12$.

[12] K. Pahlavan, X. Li, and J.-P. Makela, "Indoor geolocation science and technology," Communications Magazine, IEEE, vol. 40, no. 2, pp. 112118, Feb 2002

[13] B. J. Dil and P. J. M. Havinga, "Stochastic radio interferometric positioning in the $2.4 \mathrm{ghz}$ range," in Proceedings of the 9th ACM Conference on Embedded Networked Sensor Systems, ser. SenSys '11. New York, NY, USA: ACM, 2011, pp. 108-120.

[14] N. Patwari and A. O. Hero, III, "Using proximity and quantized rss for sensor localization in wireless networks," in Proceedings of the 2Nd ACM International Conference on Wireless Sensor Networks and Applications, ser. WSNA '03. New York, NY, USA: ACM, 2003, pp. 20-29.

[15] K. Yedavalli, B. Krishnamachari, S. Ravula, and B. Srinivasan, "Ecolocation: A sequence based technique for rf localization in wireless sensor networks," in In Proceedings of the Fourth International Symposium on Information Processing in Sensor Networks (IPSN. Inc, 2005, pp. 285-292.

[16] N. Li, B. Becerik-Gerber, B. Krishnamachari, and L. Soibelman, An Environment-Aware Sequence-Based Localization Algorithm for Supporting Building Emergency Response Operations, ch. 69, pp. 549-556.

[17] B. J. Dil and P. J. M. Havinga, "Rss-based localization with different antenna orientations," in Telecommunication Networks and Applications Conference (ATNAC), 2010 Australasian, Oct 2010, pp. 13-18.

[18] Z. Zhong, T. Zhu, D. Wang, and T. He, "Tracking with unreliable node sequences," in INFOCOM 2009, IEEE, April 2009, pp. 1215-1223.

[19] Z. Zhong and T. He, "Achieving range-free localization beyond connectivity," in Proceedings of the 7th ACM Conference on Embedded Networked Sensor Systems, ser. SenSys '09. ACM, 2009, pp. 281294.

[20] Y. Shen, W. Dai, and M. Win, "Power optimization for network localization," pp. 1-1, 2013.

[21] A. Rai, K. K. Chintalapudi, V. N. Padmanabhan, and R. Sen, "Zee: Zero-effort crowdsourcing for indoor localization," in Proceedings of the 18th Annual International Conference on Mobile Computing and Networking, ser. Mobicom '12. New York, NY, USA: ACM, 2012, pp. 293-304. 